\title{
DISTRIBUTION-FREE CALCULATION OF THE STANDARD ERROR OF CHAIN LADDER RESERVE ESTIMATES
}

\author{
By Thomas Mack \\ Munich Re, Munich
}

\begin{abstract}
A distribution-free formula for the standard error of chain ladder reserve estimates is derived and compared to the results of some parametric methods using a numerical example.
\end{abstract}

\section{KEYWORDS}

Claims reserving; chain ladder; standard error.

\section{INTRODUCTION}

The chain ladder method is probably the most popular method for estimating IBNR claims reserves. The main reason for this is its simplicity and the fact that it is distribution-free, i.e. that it seems to work with almost no assumptions. On the other hand, it is well-known that chain ladder reserve estimates for the most recent accident years are very sensitive to variations in the data observed. Moreover, in recent years many other claims reserving procedures have been proposed and the results of all these procedures vary widely and also differ more or less from the chain ladder result. Therefore it would be very helpful to know the standard error of the chain ladder reserve estimates as a measure of the uncertainty contained in the data and in order to see whether the difference between the results of the chain ladder method and any other method is significant or not.

Up to now only a few papers on claims reserving have been published which also consider the calculation of the standard error of the reserve estimate: In the papers by TAYLOR/Ashe 1983, Zehnwirth 1985, RenShaw 1989, ChrisTOFIDES 1990, VerRall 1990, VerRall 1991 essentially the same method for the calculation of the standard error is used, namely a least squares regression approach which (with the exception of Taylor/Ashe) is applied to the logarithms of the incremental claims amounts (i.e. assuming a lognormal distribution). Slightly different approaches have been proposed by WRIGHT (1990, via a generalized linear model and the method of scoring) and MACK (1991, using a gamma distribution and maximum likelihood estimation). All methods cited require a rather high amount of programming in order to calculate the many covariances between the parameter estimators. 
In the present paper, a very simple formula for the standard error of chain ladder reserve estimates is developed. The decisive step towards this formula was made by SCHNIEPER (1991). In order to calculate the rate for a casualty excess of loss cover he used a mixture of the Bornhuetter-Ferguson technique and the chain ladder method. Within this model he developed an approximation to the standard error of the estimated premium rate using a Taylor series approximation.

The present paper adapts Schnieper's idea to the claims reserving situation and contains the following additional points:

1. The model is specialized for the pure chain ladder case. This makes things easier and also makes it possible to replace the Taylor series approximation with a more exact procedure.

2. An estimate of the process variance is additionally included in the standard error of the reserve estimate. This is necessary here because the claims reserve is a random variable and not a parameter like the net premium (= expected value).

3. Schnieper intuitively claimed that the chain ladder development factors were "not strongly correlated". We prove that they are in fact uncorrelated and that therefore the reserve estimate is unbiased.

4. Besides the standard error for each accident year, a formula for the standard error of the overall reserve estimator is given, too, which takes the correlations between the estimates for the individual accident years into account.

Finally, two numerical examples are given and the results are compared to the results obtained by the aforementioned methods of Taylor/Ashe, Zehnwirth, Renshaw/Christofides, Verrall and Mack.

\section{NOTATIONS AND BASIC RESULTS}

Let $C_{i k}$ denote the accumulated total claims amount of accident year $i$, $1 \leq i \leq I$, either paid or incurred up to development year $k, 1 \leq k \leq I$. We consider $C_{i k}$ a random variable of which we have an observation if $i+k \leq I+1$ (run-off triangle). The aim is to estimate the ultimate claims amount $C_{i I}$ and the outstanding claims reserve

$$
R_{i}=C_{i I}-C_{i, I+1-i}
$$

for accident year $i=2, \ldots, I$.

The basic chain ladder assumption is that there are development factors $f_{1}, \ldots, f_{I-1}>0$ with

$$
E\left(C_{i, k+1} \mid C_{i 1}, \ldots, C_{i k}\right)=C_{i k} f_{k}, 1 \leq i \leq I, 1 \leq k \leq I-1 .
$$

The chain ladder method consists of estimating the $f_{k}$ by

$$
\hat{f}_{k}=\sum_{j=1}^{I-k} C_{j, k+1} / \sum_{j=1}^{I-k} C_{j k}, \quad 1 \leq k \leq I-1,
$$


and the ultimate claims amount $\mathrm{C}_{i I}$ by

$$
\hat{C}_{i I}=C_{i, I+1-i} \cdot \hat{f}_{I+1-i} \cdot \ldots \cdot \hat{f}_{I-1},
$$

or equivalently the reserve $R_{i}$ by

$$
\hat{R}_{i}=C_{i, I+1-i}\left(\hat{f}_{I+1-i} \cdot \ldots \cdot \hat{f}_{I-1}-1\right) .
$$

Because the chain ladder algorithm does not take into account any dependencies between accident years, we can additionally assume that the variables $C_{i k}$ of different accident years, i.e.

$$
\left\{C_{i 1}, \ldots, C_{i l}\right\},\left\{C_{j 1}, \ldots, C_{j I}\right\}, i \neq j, \text { are independent } .
$$

This must be regarded as a further implicit assumption of the chain ladder method. In practise, the independence of the accident years can be distorted by certain calendar year effects like major changes in claims handling or in case reserving.

The following theorem makes it clear that (1) and (2) are indeed the implicit assumptions of the chain ladder method.

Theorem 1: Let $D=\left\{C_{i k} \mid i+k \leq I+1\right\}$ be the set of all data observed so far. Under the assumptions (1) and (2) we have

$$
E\left(C_{i I} \mid D\right)=C_{i, I+1-i} f_{I+1-i} \cdot \ldots \cdot f_{I-1} \text {. }
$$

Proof: We use the abbreviation

$$
E_{i}(X)=E\left(X \mid C_{i 1}, \ldots, C_{i, I+1-i}\right) .
$$

Then (2) and repeated application of (1) yield

$$
\begin{aligned}
E\left(C_{i I} \mid D\right) & =E_{i}\left(C_{i I}\right) \\
& =E_{i}\left(E\left(C_{i I} \mid C_{i 1}, \ldots, C_{i, I-1}\right)\right) \\
& =E_{i}\left(C_{i, I-1} f_{I-1}\right) \\
& =E_{i}\left(C_{i, I-1}\right) f_{I-1} \\
& =\text { etc. } \\
& =E_{i}\left(C_{i, J+1-i}\right) f_{I+1-i} \cdot \ldots \cdot f_{I-1} \\
& =C_{i, I+1-i} f_{I+1-i} \cdot \ldots \cdot f_{I-1} .
\end{aligned}
$$

This theorem shows that the estimator $\hat{C}_{i I}$ has the same form as $E\left(C_{i I} \mid D\right)$ which is the best forecast of $C_{i I}$ based on the observations $D$. The next theorem shows that estimating $f_{I+1-i} \cdot \ldots \cdot f_{I-1}$ by $\hat{f}_{I+1-i} \cdot \ldots \cdot \hat{f}_{I-1}$ is indeed a reasonable procedure.

Theorem 2: Under the assumptions (1) and (2) the estimators $\hat{f}_{k}, 1 \leq k \leq I-1$, are unbiased and uncorrelated. 
Proof: Let

$$
B_{k}=\left\{C_{i j} \mid j \leq k, i+j \leq I+1\right\}, 1 \leq k \leq I .
$$

Then (2) and (1) yield

$$
E\left(C_{i, k+1} \mid B_{k}\right)=E\left(C_{i, k+1} \mid C_{i 1}, \ldots, C_{i k}\right)=C_{i k} f_{k} .
$$

We therefore have

$$
E\left(\hat{f}_{k} \mid B_{k}\right)=\sum_{j=1}^{I-k} E\left(C_{j, k+1} \mid B_{k}\right) \mid \sum_{j=1}^{I-k} C_{j k}=f_{k},
$$

which immediately gives the unbiasedness

$$
E\left(\hat{f}_{k}\right)=E\left(E\left(\hat{f}_{k} \mid B_{k}\right)\right)=f_{k}, 1 \leq k \leq I-1,
$$

of the parameter estimates. Also, the $\hat{f}_{k}$ are uncorrelated because for $j<k$

$$
\begin{aligned}
E\left(\hat{f}_{j} \hat{f}_{k}\right) & =E\left(E\left(\hat{f}_{j} \hat{f}_{k} \mid B_{k}\right)\right) \\
& =E\left(\hat{f}_{j} E\left(\hat{f}_{k} \mid B_{k}\right)\right) \\
& =E\left(\hat{f}_{j}\right) f_{k} \\
& =E\left(\hat{f}_{j}\right) E\left(\hat{f}_{k}\right) .
\end{aligned}
$$

The uncorrelatedness of the $\hat{f}_{k}$, s is surprising because $\hat{f}_{k-1}$ and $\hat{f}_{k}$ depend on the same data $C_{1 k}+\ldots+C_{I-k, k}$. The foregoing proof of the uncorrelatedness easily extends to arbitrary products of pairwise different $\hat{f}_{k}$, i.e. we have

$$
E\left(\hat{f}_{I+1-i} \cdot \ldots \cdot \hat{f}_{I-1}\right)=f_{I+1-i} \cdot \ldots \cdot f_{I-1},
$$

which shows that $\hat{C}_{i I}=C_{i, I+1-i} \hat{f}_{I+1-i} \cdot \ldots \cdot \hat{f}_{I-1}$ is an unbiased estimator of $E\left(C_{i I} \mid D\right)=C_{i, I+1-i} f_{I+1-i} \cdot \ldots \cdot f_{I-1}$. In the same way, the reserve estimator $\hat{R}_{i}$ $=\hat{C}_{i I}-C_{i, I+1-i}$ is an unbiased estimator of the true reserve $R_{i}=$ $C_{i I}-C_{i, I+1-i}$.

\section{CALCULATION OF MEAN SQUARED ERROR AND STANDARD ERROR}

The mean squared error $m s e\left(\hat{C}_{i I}\right)$ of the estimator $\hat{C}_{i I}$ of $C_{i I}$ is defined to be

$$
\operatorname{mse}\left(\hat{C}_{i I}\right)=E\left(\left(\hat{C}_{i I}-C_{i I}\right)^{2} \mid D\right)
$$

where $D=\left\{C_{i k} \mid i+k \leq I+1\right\}$ is the set of all data observed so far. Note that we are not using the unconditional mean squared error $E\left(\left(\hat{C}_{i I}-C_{i I}\right)^{2}\right)=$ $E\left(E\left(\left(\hat{C}_{i I}-C_{i I}\right)^{2} \mid D\right)\right)$ as this averages over all possible data $D$ from the underlying distribution. Instead, in practise, we are more interested in the conditional mean squared error of the particular estimated amount $\hat{C}_{i I}$ based on the specific data set $D$ observed and therefore have to use $E\left(\left(\hat{C}_{i I}-C_{i I}\right)^{2} \mid D\right)$ which just gives us the average deviation between $\hat{C}_{i I}$ and $C_{i I}$ due to future randomness only.

First, we see that

$$
\operatorname{mse}\left(\hat{R}_{i}\right)=E\left(\left(\hat{R}_{i}-R_{i}\right)^{2} \mid D\right)=E\left(\left(\hat{C}_{i I}-C_{i I}\right)^{2} \mid D\right)=\operatorname{mse}\left(\hat{C}_{i I}\right) .
$$


Next, because of the general rule $E(X-a)^{2}=\operatorname{Var}(X)+(E(X)-a)^{2}$ we have

$$
m s e\left(\hat{C}_{i l}\right)=\operatorname{Var}\left(C_{i I} \mid D\right)+\left(E\left(C_{i l} \mid D\right)-\hat{C}_{i l}\right)^{2}
$$

which shows that the mean squared error is the sum of the stochastic error (process variance) and of the estimation error.

In order to further calculate the mse we need a formula for the variance of $C_{i k}$. From the fact that $\hat{f}_{k}$ is the $C_{i k}$-weighted mean of the individual development factors $C_{i, k+1} / C_{i k}, \quad 1 \leq i \leq I-k$, we can induce that $\operatorname{Var}\left(C_{i, k+1} / C_{i k} \mid C_{i 1}, \ldots, C_{i k}\right)$ should be inversely proportional to $C_{i k}$, or equivalently

$$
\operatorname{Var}\left(C_{i, k+1} \mid C_{i 1}, \ldots, C_{i k}\right)=C_{i k} \sigma_{k}^{2}, 1 \leq i \leq I, 1 \leq k \leq I-1,
$$

with unknown parameters $\sigma_{k}^{2}, 1 \leq k \leq I-1$. This is the variance assumption which is implicitly underlying the chain ladder method.

Later on, we will need an estimator for $\sigma_{k}^{2}$. Similarly as for $\hat{f}_{k}$ it can be shown that

$$
\hat{\sigma}_{k}^{2}=\frac{1}{I-k-1} \sum_{i=1}^{I-k} C_{i k}\left(\frac{C_{i, k+1}}{C_{i k}}-\hat{f}_{k}\right)^{2}, \quad 1 \leq k \leq I-2 .
$$

is an unbiased estimator of $\sigma_{k}^{2}, 1 \leq k \leq I-2$. We still lack an estimator for $\sigma_{I-1}$. If $\hat{f}_{I-1}=1$ and if the claims development is believed to be finished after $I-1$ years, we can put $\hat{\sigma}_{I-1}=0$. If not, we extrapolate the usually exponentially decreasing series $\hat{\sigma}_{1}, \ldots, \hat{\sigma}_{I-3}, \hat{\sigma}_{I-2}$ by one additional member, for instance by loglinear regresssion or more simply by requiring that

$$
\hat{\sigma}_{I-3} / \hat{\sigma}_{I-2}=\hat{\sigma}_{I-2} / \hat{\sigma}_{I-1}
$$

holds at least as long as $\hat{\sigma}_{I-3}>\hat{\sigma}_{I-2}$. This last possibility leads to

$$
\hat{\sigma}_{I-1}^{2}=\min \left(\hat{\sigma}_{I-2}^{4} / \hat{\sigma}_{I-3}^{2}, \min \left(\hat{\sigma}_{I-3}^{2}, \hat{\sigma}_{I-2}^{2}\right)\right)
$$

which has been used in the examples.

Now, we are able to state and prove the main result

Theorem 3: Under the assumptions (1), (2) and (3) the mean squared error mse $\left(\hat{R}_{i}\right)$ can be estimated by

$$
\widehat{m s e\left(\hat{R}_{i}\right)}=\hat{C}_{i I}^{2} \sum_{k=I+1-i}^{I-1} \frac{\hat{\sigma}_{k}^{2}}{\hat{f}_{k}^{2}}\left(\frac{1}{\hat{C}_{i k}}+\frac{1}{\sum_{j=1}^{I-k} C_{j k}}\right)
$$

where $\hat{C}_{i k}=C_{i, I+1-i} \hat{f}_{I+1-i} \cdot \ldots \cdot \hat{f}_{k-1}, k>I+1-i$, are the estimated values of the future $C_{i k}$ and $\hat{C}_{i, I+1-i}=C_{i, I+1-i}$. 
Proof: We use the abbreviations

$$
\begin{aligned}
E_{i}(X) & =E\left(X \mid C_{i 1}, \ldots, C_{i, I+1-i}\right), \\
\operatorname{Var}_{i}(X) & =\operatorname{Var}\left(X \mid C_{i 1}, \ldots, C_{i, I+1-i}\right) .
\end{aligned}
$$

We start from

$$
\operatorname{mse}\left(\hat{R}_{i}\right)=\operatorname{Var}\left(C_{i I} \mid D\right)+\left(E\left(C_{i I} \mid D\right)-\hat{C}_{i I}\right)^{2} .
$$

Repeated application of the basic chain ladder assumption (1) and of the above variance assumption (3) yields for the first term of $m s e\left(\hat{R}_{i}\right)$

$$
\begin{aligned}
\operatorname{Var}\left(C_{i I} \mid D\right)= & \operatorname{Var}_{i}\left(C_{i I}\right) \\
= & E_{i}\left(\operatorname{Var}\left(C_{i I} \mid C_{i 1}, \ldots, C_{i, I-1}\right)\right)+ \\
& +\operatorname{Var}_{i}\left(E\left(C_{i I} \mid C_{i 1}, \ldots, C_{i, I-1}\right)\right) \\
= & E_{i}\left(C_{i, I-1}\right) \sigma_{I-1}^{2}+\operatorname{Var}_{i}\left(C_{i, I-1}\right) f_{I-1}^{2} \\
= & E_{i}\left(C_{i, I-2}\right) f_{I-2} \sigma_{I-1}^{2}+E_{i}\left(C_{i, I-2}\right) \sigma_{I-2}^{2} f_{I-1}^{2}+ \\
& +\operatorname{Var}_{i}\left(C_{i, I-2}\right) f_{I-2}^{2} f_{I-1}^{2} \\
= & \operatorname{etc.} \\
= & C_{i, I+1-i} \sum_{k=I+1-i}^{I-1} f_{I+1-i} \ldots f_{k-1} \sigma_{k}^{2} f_{k+1}^{2} \ldots f_{I-1}^{2}
\end{aligned}
$$

because of $\operatorname{Var}_{i}\left(C_{i, 1+1-i}\right)=0$.

Due to Theorem 1 we obtain for the second term of mse $\left(\hat{R}_{i}\right)$

$$
\left(E\left(C_{i I} \mid D\right)-\hat{C}_{i I}\right)^{2}=C_{\mathrm{i}, I+1-i}^{2}\left(f_{I+1-\mathrm{i}} \cdot \ldots \cdot f_{I-1}-\hat{f}_{I+1-i} \cdot \ldots \cdot \hat{f}_{I-1}\right)^{2} .
$$

In practice, we must find estimators for these two terms of $m s e\left(\hat{R}_{i}\right)$. For the first term this will be done by replacing the unknown parameters $f_{k}$ and $\sigma_{k}^{2}$ with their estimators $\hat{f}_{k}$ and $\hat{\sigma}_{k}^{2}$, i.e. we estimate $\operatorname{Var}\left(C_{i I} \mid D\right)$ by

$$
\begin{gathered}
C_{i, I+1-i}\left(\sum_{k=I+1-i}^{I-1} \hat{f}_{I+1-i} \cdot \ldots \cdot \hat{f}_{k-1} \cdot \sigma_{k}^{2} \cdot \hat{f}_{k+1}^{2} \cdot \ldots \cdot \hat{f}_{I-1}^{2}\right) \\
=\hat{C}_{i I}^{2} \sum_{k=I+1-i}^{I-1} \frac{\hat{\sigma}_{k}^{2} / \hat{f}_{k}^{2}}{\hat{C}_{i k}}
\end{gathered}
$$

where we have used the notation $\hat{C}_{i k}$ introduced in the theorem.

But in the second term $\left(^{*}\right)$ of $m s e\left(\hat{R}_{i}\right)$ we can not simply replace $f_{k}$ with $\hat{f}_{k}$ because this would yield 0 . We therefore use a different approach. We can write

$$
\begin{aligned}
F & =f_{I+1-i} \cdot \ldots \cdot f_{I-1}-\hat{f}_{I+1-i} \cdot \ldots \cdot \hat{f}_{I-1} \\
& =S_{I+1-i}+\ldots+S_{I-1}
\end{aligned}
$$

with

$$
S_{k}=\hat{f}_{I+1-i} \cdot \ldots \cdot \hat{f}_{k-1}\left(f_{k}-\hat{f}_{k}\right) f_{k+1} \cdot \ldots \cdot f_{I-1}
$$


and therefore

$$
\begin{aligned}
F^{2} & =\left(S_{I+1-i}+\ldots+S_{I-1}\right)^{2} \\
& =\sum_{k=I+1-i}^{I-1} S_{k}^{2}+2 \sum_{j<k} S_{j} S_{k} .
\end{aligned}
$$

Now we replace $S_{k}^{2}$ with $E\left(S_{k}^{2} \mid B_{k}\right)$ and $S_{j} S_{k}, j<k$, with $E\left(S_{j} S_{k} \mid B_{k}\right)$. This means that we approximate $S_{k}^{2}$ and $S_{j} S_{k}$ by averaging over as little data as possible such that as many values $C_{i k}$ as possible from the observed data are kept fixed. Because of $E\left(f_{k}-\hat{f}_{k} \mid B_{k}\right)=0$ (see the proof of Theorem 2) we obtain $E\left(S_{j} S_{k} \mid B_{k}\right)=0$ for $j<k$. Because of

$$
\begin{aligned}
E\left(\left(f_{k}-\hat{f}_{k}\right)^{2} \mid B_{k}\right) & =\operatorname{Var}\left(\hat{f}_{k} \mid B_{k}\right) \\
& =\sum_{j=1}^{I-k} \operatorname{Var}\left(C_{j, k+1} \mid B_{k}\right) \mid\left(\sum_{j=1}^{I-k} C_{j k}\right)^{2} \\
& =\sigma_{k}^{2} / \sum_{j=1}^{I-k} C_{j k}
\end{aligned}
$$

we obtain

$$
E\left(S_{k}^{2} \mid B_{k}\right)=\hat{f}_{I+1-i}^{2} \cdot \ldots \cdot \hat{f}_{\mathrm{k}-1}^{2} \sigma_{k}^{2} f_{k+1}^{2} \cdot \ldots \cdot f_{I-1}^{2} / \sum_{i=1}^{I-k} C_{i k}
$$

Taken together, we replace $F^{2}=\left(\Sigma S_{k}\right)^{2}$ with $\Sigma_{k} E\left(S_{k}^{2} \mid B_{k}\right)$ and because all terms of this sum are positive we now can replace all unknown parameters $f_{k}$, $\sigma_{k}^{2}$ with their unbiased estimators $\hat{f}_{k}, \sigma_{k}^{2}$. Altogether, we estimate $F^{2}=$ $\left(f_{I+1-i} \cdot \ldots \cdot f_{I-1}-\hat{f}_{I+1-i} \cdot \ldots \cdot \hat{f}_{I-1}\right)^{2}$ by

$$
\begin{gathered}
\sum_{k=I+1-i}^{I-1}\left(\hat{f}_{I+1-i}^{2} \cdot \ldots \cdot \hat{f}_{k-1}^{2} \cdot \hat{\sigma}_{k}^{2} \cdot \hat{f}_{k+1}^{2} \cdot \ldots \cdot \hat{f}_{I-1}^{2} \mid \sum_{j=1}^{I-k} C_{j k}\right) \\
=\hat{f}_{I+1-i}^{2} \cdot \ldots \cdot \hat{f}_{I-1}^{2} \sum_{k=I+1-i}^{I-1} \frac{\hat{\sigma}_{k}^{2} / \hat{f}_{k}^{2}}{\sum_{j=1}^{I-k} C_{j k}}
\end{gathered}
$$

This finally leads to the estimator stated in the theorem.

The square root s.e. $\left(\hat{R}_{i}\right)$ of an estimator of the mean squared error is defined to be the standard error of $\hat{R}_{i}$.

Often the standard error of the overall reserve estimate $\hat{R}=\hat{R}_{2}+\ldots+\hat{R}_{I}$ is of interest, too. In this case we cannot simply add together the values of (s.e. $\left.\left(\hat{R}_{i}\right)\right)^{2}, 2 \leq i \leq 1$, because they are correlated via the common estimators $\hat{f}_{k}$ and $\hat{\sigma}_{k}$. We therefore proceed as before and obtain: 
Corollary: With the assumptions and notations of Theorem 3 the mean squared error of the overall reserve estimate $\hat{R}=\hat{R}_{2}+\ldots+\hat{R}_{I}$ can be estimated by

$$
\widehat{m s e(\hat{R})}=\sum_{i=2}^{I}\left\{\left(\text { s.e. }\left(\hat{R}_{i}\right)\right)^{2}+\hat{C}_{i I}\left(\sum_{j=i+1}^{I} \hat{C}_{j I}\right) \sum_{k=I+1-i}^{I-1} \frac{2 \hat{\sigma}_{k}^{2} / \hat{f}_{k}^{2}}{\sum_{n=1}^{I-k} C_{n k}}\right\}
$$

Proof: We have

$$
\begin{aligned}
\text { mse }\left(\sum_{i=2}^{I} \hat{R}_{i}\right) & =E\left(\left(\sum_{i=2}^{I} \hat{R}_{i}-\sum_{i=2}^{I} R_{i}\right)^{2} \mid D\right) \\
& =E\left(\left(\sum_{i=2}^{I} \hat{C}_{i I}-\sum_{i=2}^{I} C_{i I}\right)^{2} \mid D\right) \\
& =\operatorname{Var}\left(\sum_{i=2}^{I} C_{i I} \mid D\right)+\left(E\left(\sum_{i=2}^{I} C_{i I} \mid D\right)-\sum_{i=2}^{I} \hat{C}_{i I}\right)^{2} .
\end{aligned}
$$

The independence of the accident years yields

$$
\operatorname{Var}\left(\sum_{i=2}^{I} C_{i l} \mid D\right)=\sum_{i=2}^{I} \operatorname{Var}\left(C_{i I} \mid D\right),
$$

whose summands have already been calculated in the proof of Theorem 3 . Furthermore

$$
\begin{aligned}
\left(E\left(\sum_{i=2}^{I} C_{i I} \mid D\right)-\sum_{i=2}^{I} \hat{C}_{i I}\right)^{2} & =\left(\sum_{i=2}^{I}\left(E\left(C_{i I} \mid D\right)-\hat{C}_{i I}\right)\right)^{2} \\
& =\sum_{i, j}\left(E\left(C_{i I} D\right)-\hat{C}_{i I}\right) \cdot\left(E\left(C_{j I} \mid D\right)-\hat{C}_{j I}\right) \\
& =\sum_{i, j} C_{i, I+1-i} C_{j, I+1-j} F_{i} F_{j}
\end{aligned}
$$

with

Observing

$$
F_{i}=f_{I+1-i} \ldots f_{I-1}-\hat{f}_{I+1-i} \ldots \hat{f}_{I-1}
$$

$$
m s e\left(\hat{R}_{i}\right)=\operatorname{Var}\left(C_{i I} \mid D\right)+\left(C_{i, 1+1-i} F_{i}\right)^{2}
$$

(cf. $\left({ }^{*}\right)$ in the proof of theorem 3 ) we see that

$$
m s e\left(\sum_{i=2}^{I} \hat{R}_{i}\right)=\sum_{i=2}^{I} \operatorname{mse}\left(\hat{R}_{I}\right)+\sum_{2 \leq i<j \leq I} 2 \cdot C_{i, I+1-i} C_{j, I+1-j} F_{i} F_{j} .
$$


An analogous procedure as for $F^{2}$ in the above proof yields for $F_{i} F_{j}, i<j$, the estimator

$$
\sum_{k=I+1-i}^{I-1} \hat{f}_{I+1-j} \cdot \ldots \cdot \hat{f}_{I-i} \hat{f}_{I+1-i}^{2} \cdot \ldots \cdot \hat{f}_{k-1}^{2} \hat{\sigma}_{k}^{2} \hat{f}_{k+1}^{2} \cdot \ldots \cdot \hat{f}_{I-1}^{2} / \sum_{n=1}^{I-k} C_{n k}
$$

This completes the proof.

\section{EXAMPLES}

In the first example we use the TAYLOR/AsHE (1983) data, which were also used by VerRall $(1990,1991)$.

TABLE 1

RUN-OFF TRIANGLE (ACCUMULATED FIGURES)

\begin{tabular}{|c|c|c|c|c|c|c|c|c|c|c|}
\hline$i$ & $C_{i 1}$ & $C_{i 2}$ & $C_{i 3}$ & $C_{i 4}$ & $C_{i s}$ & $C_{i 6}$ & $C_{i 7}$ & $C_{i 8}$ & $C_{\theta \theta}$ & $C_{i 10}$ \\
\hline 1 & 357848 & 1124788 & 1735330 & 2218270 & 2745596 & 3319994 & 3466336 & 3606286 & 3833515 & 3901463 \\
\hline 2 & 352118 & 1236139 & 2170033 & 3353322 & 3799067 & 4120063 & 4647867 & 4914039 & 5339085 & \\
\hline 3 & 290507 & 1292306 & 2218525 & 3235179 & 3985995 & 4132918 & 4628910 & 4909315 & & \\
\hline 4 & 310608 & 1418858 & 2195047 & 3757447 & 4029929 & 4381982 & 4588268 & & & \\
\hline 5 & 443160 & 1136350 & 2128333 & 2897821 & 3402672 & 3873311 & & & & \\
\hline 6 & 396132 & 1333217 & 2180715 & 2985752 & 3691712 & & & & & \\
\hline 7 & 440832 & 1288463 & 2419861 & 3483130 & & & & & & \\
\hline 8 & 359480 & 1421128 & 2864498 & & & & & & & \\
\hline 9 & 376686 & 1363294 & & & & & & & & \\
\hline 10 & 344014 & & & & & & & & & \\
\hline
\end{tabular}

This yields the following parameter estimates $(k=1, \ldots, 9)$ :

$\hat{f}_{k}: 3.49,1.75,1.46,1.174,1.104,1.086,1.054,1.077,1.018$

$\hat{\sigma}_{k}^{2} / 1000: 160,37.7,42.0,15.2,13.7,8.19,0.447,1.15,0.477$

TABLE 2

ESTIMATED RESERVES $\hat{R}_{i}$ IN $1000 \mathrm{~s}$

\begin{tabular}{lrrrrrr}
\hline \hline & $\begin{array}{c}\text { Chain } \\
\text { ladder }\end{array}$ & $\begin{array}{c}\text { Verrall } \\
1991\end{array}$ & $\begin{array}{c}\text { Renshaw } \\
\text { Christofides }\end{array}$ & Zehnwirth & $\begin{array}{c}\text { Mack } \\
1991\end{array}$ & $\begin{array}{c}\text { Taylor } \\
\text { Ashe }\end{array}$ \\
\hline$i=2$ & 95 & 96 & 111 & 109 & 93 & 298 \\
$i=3$ & 470 & 439 & 482 & 473 & 447 & 600 \\
$i=4$ & 710 & 608 & 661 & 648 & 611 & 745 \\
$i=5$ & 985 & 1011 & 1091 & 1069 & 992 & 1077 \\
$i=6$ & 1419 & 1423 & 1531 & 1500 & 1453 & 1788 \\
$i=7$ & 2178 & 2150 & 2311 & 2265 & 2186 & 2879 \\
$i=8$ & 3920 & 3529 & 3807 & 3731 & 3665 & 4221 \\
$i=9$ & 4279 & 4056 & 4452 & 4364 & 4122 & 4866 \\
$i=10$ & 4626 & 4340 & 5066 & 4965 & 4516 & 5827 \\
\hline overall & 18681 & 16652 & 19512 & 19124 & 18085 & 22301 \\
\hline
\end{tabular}


TABLE 3

STANDARD ERROR IN $\%$ OF $\hat{R}_{i}$

\begin{tabular}{lcccccc}
\hline \hline & $\begin{array}{c}\text { Chain } \\
\text { ladder }\end{array}$ & $\begin{array}{c}\text { Verrall } \\
1991\end{array}$ & $\begin{array}{c}\text { Renshaw } \\
\text { Christofides }\end{array}$ & Zehnwirth & $\begin{array}{c}\text { Mack } \\
1991\end{array}$ & $\begin{array}{c}\text { Taylor } \\
\text { Ashe }\end{array}$ \\
\hline$i=2$ & $80 \%$ & $49 \%$ & $54 \%$ & $49 \%$ & $40 \%$ & $27 \%$ \\
$i=3$ & $26 \%$ & $37 \%$ & $39 \%$ & $35 \%$ & $30 \%$ & $20 \%$ \\
$i=4$ & $19 \%$ & $30 \%$ & $32 \%$ & $29 \%$ & $24 \%$ & $18 \%$ \\
$i=5$ & $27 \%$ & $27 \%$ & $28 \%$ & $25 \%$ & $21 \%$ & $16 \%$ \\
$i=6$ & $29 \%$ & $25 \%$ & $26 \%$ & $24 \%$ & $20 \%$ & $16 \%$ \\
$i=7$ & $26 \%$ & $25 \%$ & $26 \%$ & $24 \%$ & $20 \%$ & $14 \%$ \\
$i=8$ & $22 \%$ & $27 \%$ & $28 \%$ & $26 \%$ & $21 \%$ & $14 \%$ \\
$i=9$ & $23 \%$ & $30 \%$ & $31 \%$ & $30 \%$ & $24 \%$ & $14 \%$ \\
\hline$i=10$ & $29 \%$ & $38 \%$ & $40 \%$ & $39 \%$ & $31 \%$ & $14 \%$ \\
\hline overall & $13 \%$ & $15 \%$ & $16 \%$ & $16 \%$ & & $9 \%$ \\
\hline
\end{tabular}

\section{Comments :}

The results for 'Taylor/Ashe' and 'Verrall 1991' have been taken from these papers. Taylor/Ashe produced much lower standard errors than the other methods. This is due to the fact that their reserve estimates employed only 6 parameters (as compared to 19 of the other methods) and that they additionally used the information on the numbers of claims finalized.

Renshaw and Christofides describe the same loglinear regression method which is also identical to Verrall's (1990) Bayesian approach without any prior information. Therefore the results for 'Renshaw/Christofides' have been taken from VerRall (1990), Table 2.

The results for 'Zehnwirth' have been obtained by using his ICRFS software package version 6.1 employing one of his fixed parameter development factor models which he calls 'chain ladder model'. We have used it without any further adjustment. It should be remarked that this is not what Zehnwirth intends, as his software package is a modelling framework and any initial model should be further adjusted interactively with the help of the indications and plots given by the program. Without any further adjustment this 'chain ladder model' is identical to the Renshaw/Christofides model, i.e. it is a loglinearized approximation of the usual chain ladder model. The fact that it leads to slightly lower results is attributable to using a different estimator for the model variance.

The results for 'Mack 1991' have been obtained according to a previous paper (MACK (1991)) of the author but additionally an estimate of the process variance has been included, as this is the case with all the other methods.

The estimated reserves of all methods except 'Taylor/Ashe' differ by less than $20 \%$ and are therefore according to Table 3 within one standard error. For the chain ladder method neither the reserve estimates nor the standard errors are systematically higher or lower than for the other methods (except 'Taylor/Ashe'). The reason for the comparatively high chain ladder standard 
error of $80 \%$ for accident year 2 is the fact that the reserve $\hat{R}_{2}$ itself is very low in comparison to the other reserves $\hat{R}_{3}, \ldots, \hat{R}_{10}$ : If we look at the sequence $\hat{R}_{10}, \hat{R}_{9}, \ldots, \hat{R}_{4}, \hat{R}_{3}$ we see that $\hat{R}_{i-1}$ is always greater then $\hat{R}_{i} / 2$ but $\hat{R}_{2}$ is smaller than $\hat{R}_{3} / 4$. This fact is very well reflected by the high standard error of $80 \%$.

A closer look at the Taylor/Ashe data shows that the individual development factors $C_{i, k+1} / C_{i k}, 1 \leq i \leq I-k$, do not fluctuate much around their mean value $\hat{f}_{k}$ so that the whole triangle can be considered as relatively regular. Therefore Taylor/Ashe were able to dispense with taking logarithms and thus avoided the problem of transforming back the result into the original data space. We therefore give a second example, which is less regular and where the claims amounts of the most recent accident years are much lower than in the previous years. These data (mortgage guarantee business) were compiled from a competition presented by SANDERS (1990).

TABLE 4

RUN-OFF TRIANGLE (ACCUMULATED FIGURES)

\begin{tabular}{|c|c|c|c|c|c|c|c|c|c|}
\hline$i$ & $C_{i 1}$ & $C_{i 2}$ & $C_{i 3}$ & $C_{i 4}$ & $C_{i 5}$ & $C_{i 6}$ & $C_{i 7}$ & $C_{i 8}$ & $C_{i 9}$ \\
\hline 1 & 58046 & 127970 & 476599 & 1027692 & 1360489 & 1647310 & 1819179 & 1906852 & 1950105 \\
\hline 2 & 24492 & 141767 & 984288 & 2142656 & 2961978 & 3683940 & 4048898 & 4115760 & \\
\hline 3 & 32848 & 274682 & 1522637 & 3203427 & 4445927 & 5158781 & 5342585 & & \\
\hline 4 & 21439 & 529828 & 2900301 & 4999019 & 6460112 & 6853904 & & & \\
\hline 5 & 40397 & 763394 & 2920745 & 4989572 & 5648563 & & & & \\
\hline 6 & 90748 & 951994 & 4210640 & 5866482 & & & & & \\
\hline 7 & 62096 & 868480 & 1954797 & & & & & & \\
\hline 8 & 24983 & 284441 & & & & & & & \\
\hline 9 & 13121 & & & & & & & & \\
\hline
\end{tabular}

Parameter estimates $(k=1, \ldots, 8)$ :

$\hat{f}_{k}: 11.1,4.09,1.71,1.28,1.14,1.069,1.026,1.023$

$\hat{\sigma}_{k}^{2} / 1000: 1787,977,194,42.8,27.0,5.57,1.26,0.285$

TABLE 5

ESTIMATED RESERVES $\hat{R}_{i}$ IN $1000 \mathrm{~s}$

\begin{tabular}{|c|c|c|c|c|}
\hline & $\begin{array}{l}\text { Chain } \\
\text { ladder }\end{array}$ & $\begin{array}{c}\text { Renshaw } \\
\text { Christofides }\end{array}$ & Zehnwirth & $\begin{array}{c}\text { Mack } \\
1991\end{array}$ \\
\hline$i=2$ & 93 & 91 & 87 & 62 \\
\hline$i=3$ & 265 & 275 & 262 & 199 \\
\hline$i=4$ & 834 & 818 & 778 & 682 \\
\hline$i=5$ & 1568 & 1979 & 1884 & 1639 \\
\hline$i=6$ & 3696 & 5497 & 5231 & 4420 \\
\hline$i=7$ & 3487 & 6650 & 6328 & 5378 \\
\hline$i==8$ & 2956 & 4331 & 4122 & 3143 \\
\hline$i=9$ & 1647 & 2339 & 2226 & 1555 \\
\hline overall & 14547 & 21980 & 20919 & 17078 \\
\hline
\end{tabular}


TABLE 6

STANDARD ERROR IN $\%$ OF $\hat{R}_{i}$

\begin{tabular}{lcccc}
\hline & $\begin{array}{c}\text { Chain } \\
\text { ladder }\end{array}$ & $\begin{array}{c}\text { Renshaw } \\
\text { Christofides }\end{array}$ & Zehnwirth & $\begin{array}{c}\text { Mack } \\
1991\end{array}$ \\
\hline$i=2$ & $65 \%$ & $90 \%$ & $80 \%$ & $60 \%$ \\
$i=3$ & $53 \%$ & $60 \%$ & $53 \%$ & $41 \%$ \\
$i=4$ & $38 \%$ & $51 \%$ & $45 \%$ & $37 \%$ \\
$i=5$ & $38 \%$ & $48 \%$ & $42 \%$ & $35 \%$ \\
$i=6$ & $28 \%$ & $46 \%$ & $41 \%$ & $33 \%$ \\
$i=7$ & $37 \%$ & $47 \%$ & $42 \%$ & $34 \%$ \\
$i=8$ & $61 \%$ & $50 \%$ & $47 \%$ & $36 \%$ \\
$i=9$ & $133 \%$ & $66 \%$ & $64 \%$ & $47 \%$ \\
\hline overall & $26 \%$ & & $24 \%$ & \\
\hline
\end{tabular}

Here all results have been calculated by the author. In comparison with the standard errors of the first example, the chain ladder standard errors now reflect very well the generally higher uncertainty of this second triangle and especially the uncertainty of the last two accident years where the relative standard errors are very high because the reserve estimates are comparatively low. The most extreme deviation between the reserve estimates of the different methods is for accident year 7 where the 'Renshaw/Christofides' reserve exceeds the chain ladder reserve by 2.5 standard errors.

Altogether, if the impressions of these two examples can be taken as typical, we can conclude that the standard errors are of about the same size for the chain ladder as with the other methods, although they do not show such a smooth pattern as these because the other methods use only one $\sigma^{2}$ parameter as compared to $I-1$ of chain ladder. But this could also be achieved for the chain ladder method by smoothing out the $\hat{\sigma}_{k}^{2}$ s by means of an exponential function $\exp (a-b k)$.

Finally, we must bear in mind that these standard errors can only reflect the estimation error and the statistical error, but not the specification error, i.e. the fact that the model chosen can be wrong or that the future development may not be in accordance with past experience.

\section{ACKNOWLEDGEMENT}

I am indebted to AloIs GISLER for pointing out the correct definition of the mean squared error and some further useful remarks.

\section{REFERENCES}

Christofides, S. (1990) Regression Models Based on Logincremental Payments. In: Claims Reserving Manual, Vol. 2, Institute of Actuaries, London.

MACK, Th. (1991) A Simple Parametric Model for Rating Automobile Insurance or Estimating IBNR Claims Reserves. ASTIN Bulletin 21, 93-109.

Renshaw, A. (1989) Chain Ladder and Interactive Modelling. Journal of the Institute of Actuaries 116, 559-587. 
Sanders, D. E. A. (1990) Competition Presented at a London Market Actuaries Dinner.

SCHNIEPER, R. (1991) Separating True IBNR and IBNER Claims. ASTIN Bulletin 21, 111-127.

TAYlor, G.C. and ASHe, F.R. (1983) Second Moments of Estimates of Outstanding Claims. Journal of Econometrics 23, 37-61.

Verrall, R.J. (1990) Bayes and Empirical Bayes Estimation for the Chain Ladder Model. ASTIN-Bulletin 20, 217-243.

Verrall, R. J. (1991) On the Estimation of Reserves from Loglinear Models. Insurance: Mathematics and Economics 10, 75-80.

Wright, T.S. (1990) A Stochastic Method for Claims Reserving in General Insurance. Journal of the Institute of Actuaries 117, 677-731.

Zehnwirth, B. ( $\geq 1985$ ) Interactive Claims Reserving Forecasting System. Insureware P/L, E. St. Kilda Vic 3183, Australia.

\section{Thomas MACK}

Münchener Rückversicherungs-Gesellschaft, Königinstrasse 107, D-80791 München. 